Article

\title{
Modelling and Stability Analysis of Wind Power Plants Connected to Weak Grids
}

\author{
Esmaeil Ebrahimzadeh ${ }^{1}$, Frede Blaabjerg ${ }^{2, *} \mathbb{D}$, Torsten Lund ${ }^{1}$, John Godsk Nielsen ${ }^{1}$ and \\ Philip Carne Kjær ${ }^{1}$ \\ 1 Vestas Wind Systems, 8200 Aarhus N, Denmark; esebr@vestas.com (E.E.); tolud@vestas.com (T.L.); \\ jogn@vestas.com (J.G.N.); pck@vestas.com (P.C.K.) \\ 2 Department of Energy Technology, Aalborg University, 9220 Aalborg, Denmark \\ * Correspondence: fbl@et.aau.dk; Tel.: +45-2129-2454
}

Received: 30 August 2019; Accepted: 29 October 2019; Published: 4 November 2019

Featured Application: A unified frequency-domain modelling and stability analysis is presented to predict the impact of mutual interactions between a wind power plant and a weak grid on the stability margin.

\begin{abstract}
It is important to develop modelling tools to predict unstable situations resulting from the interactions between the wind power plant and the weak power system. This paper presents a unified methodology to model and analyse a wind power plant connected to weak grids in the frequency-domain by considering the dynamics of the phase lock loop (PLL) and controller delays, which have been neglected in most of the previous research into modelling of wind power plants to simplify modelling. The presented approach combines both $\mathrm{dq}$ and positive/negative sequence domain modelling, where a single wind turbine is modelled in the dq domain but the whole wind power plant connected to the weak grid is analysed in the positive/negative sequence domain. As the proposed modelling of the wind power plant is systematic and modular and based on the decoupled positive/negative sequence impedances, the application of the proposed methodology is relevant for transmission system operators (TSOs) to assess stability easily with a very low compactional burden. In addition, as the analytical dq impedance models of the single wind turbine are provided, the proposed methodology is an optimization design tool permitting wind turbine manufacturers to tune their converter control. As a case study, a $108 \mathrm{MW}$ wind power plant connected to a weak grid was used to study its sensitivity to variations in network short-circuit level, $\mathrm{X} / \mathrm{R}$ ratio and line series capacitor compensation $\left(\mathrm{Xc}_{\mathrm{c}} \mathrm{Xg}\right)$.
\end{abstract}

Keywords: wind power plants; wind turbines; weak grids; impedance-based modelling; small-signal stability

\section{Introduction}

With the increasing number of solar and wind power plants in the electrical grid, the installation ratio of traditional synchronous generators to the total production capacity is significantly decreasing, which is leading to weak transmission grids [1]. Worldwide, the transmission system operators are in the process of updating their grid connection requirements for generation units to ensure that transmission networks can continue to be operated in a secure manner when renewable energy resources replace traditional thermal generation units. It is important to have simulation models, which can reliably predict the stability margins under contemporary operational conditions. The grid-stability of such systems can be improved in multiple ways through combinations of properties of the generation units and an additional balance of plant equipment such as STATCOMS, battery storage, etc. 
In the literature, time-domain analysis [2,3] and frequency-domain analysis [4,5] have been used to model and analyse different power electronics-based power systems. Linearised frequency-domain analysis is accurate around an operating point for an intended frequency range. It requires much less computational power than non-linear time-domain simulations [6]. State-space modelling and impedance-based modelling are two popular approaches in the frequency-domain $[7,8]$. In these approaches, the stability can be analysed based on poles, eigenvalues, Nyquist plots or Bode plots. So far, state-space and impedance-based modelling have been used in different power electronics-based power systems like microgrids, wind power plants, solar plants, parallel current source converters and voltage source inverters. In the following section, papers, which are based on the state-space and impedance-based methods, are reviewed.

In general, there are three methods for state-space modelling: state-space averaging method, harmonic state-space (HSS) method and component connection method (CCM). State-space averaging modelling is a basic method to model and analyse a dynamic system. In this method, a linearised system is presented by the state variable matrix, input matrix and output matrix. In the literature, various power electronic systems like wind power plants and microgrids have been modelled based on this approach $[9,10]$. However, as this method is not modular, it can be complex for large-scale power electronic systems [11]. In the linear time-periodic (LTP) systems, where there are couplings between different frequencies, a powerful analysis tool is the harmonic state-space (HSS) method [12,13]. The harmonic state-space model of an LTP system can generally be written based on the Fourier coefficients of the state matrices [13]. Compared to the state-space averaging method, the harmonic state-space method is more accurate but also more complex, as this approach models the frequency-couplings in the system. In the component connection method (CCM), first a dynamic system is divided into some smaller subsystems and each subsystem is modelled based on the state-space method. Then, all individual matrices will be placed in new diagonal matrices. For large-scale dynamic systems, CCM is much more systematic and modular compared to the state-space averaging method [14-16].

In addition to state-space modelling, impedance-based modelling is another useful tool for frequency-domain analysis [17]. In this method, the grid output impedance (Zs) and the converter input impedance $(\mathrm{Zl})$ are required for stability analysis $[5,18]$. The impedance/admittance matrices can be calculated in the dq domain or in the sequence domain. In the sequence domain, by perturbing a three-phase power converter at a frequency $\mathrm{fp}+\mathrm{f} 1$ in the positive sequence and a frequency $\mathrm{fp}-$ $\mathrm{f} 1$ in the negative sequence, the small-signal relationship between the output current and voltages can be written by a $2 \times 2$ impedance/admittance matrix [19]. As the coupling between negative and positive sequence is very small, off-diagonal elements of the impedance matrix can be neglected for stability analysis $[5,20]$. In the dq domain, the three-phase converter is disturbed in the dq frame, where three-phase variables can be transformed into the dq domain by the Park's transformation. Similar to the sequence domain, the small-signal impedance/admittance of the converter can be written by a $2 \times 2$ impedance/admittance matrix [21,22]. In the dq domain, there is a high-coupling between $\mathrm{d}$-axis and q-axis. Therefore, for the stability analysis, off-diagonal impedance matrix elements cannot be neglected like they can in the sequence domain [23-25]. As the converter controller is usually implemented in the $\mathrm{dq}$ frame, $\mathrm{dq}$ domain modelling is simpler and more efficient for the controller design compared to the sequence domain modelling. However, for a stability analysis of the whole system, dq domain impedance-based modelling is a more complex method because of high-coupling between the d-axis and q-axis. As the impedance data of the grid is usually available in the positive/negative sequence domain and the sequence domain impedance matrix is almost decupled, it is more efficient to do a stability analysis of a large system in the sequence domain. In order to have both the advantages of $\mathrm{dq}$ domain and sequence domain modelling, this paper presents a systematic and modular method, where the wind turbine is modelled in the $\mathrm{dq}$ domain and where stability analysis of the wind power plant is analysed in the sequence domain. In fact, for stability analysis, dq domain impedance is transferred to the sequence domain using a transformation matrix. The equivalent positive sequence impedance 
is used to analyse the wind power plant stability based on the system poles and based on Nyquist criterion of the open-loop system. The presented method is scalable and adaptable to different scales of the power system along with a low computational burden.

In the following section, a wind turbine with full-scale converter configuration is considered to be modelled. The dynamics of the PLL and the time delay, which have been usually neglected in the literature on wind power plant stability analysis [24], are also included to have a more accurate model. Then, a $108 \mathrm{MW}$ wind power plant connected to a weak grid is analysed under different conditions.

\section{Full-Scale Converter-Based Wind Turbines}

The full-scale converter configuration, which is shown in Figure 1, is one of the popular configurations for variable speed wind turbines. In this configuration, the grid side converter usually controls the dc-link voltage, and the machine side converter controls the active power [26]. If the small oscillations of the dc-link voltage are neglected, the dynamics of the wind turbine around and above the fundamental frequency can be predicted by modelling of the grid side converter [27]. Therefore, the dynamics of the machine side converter can be neglected in this paper.

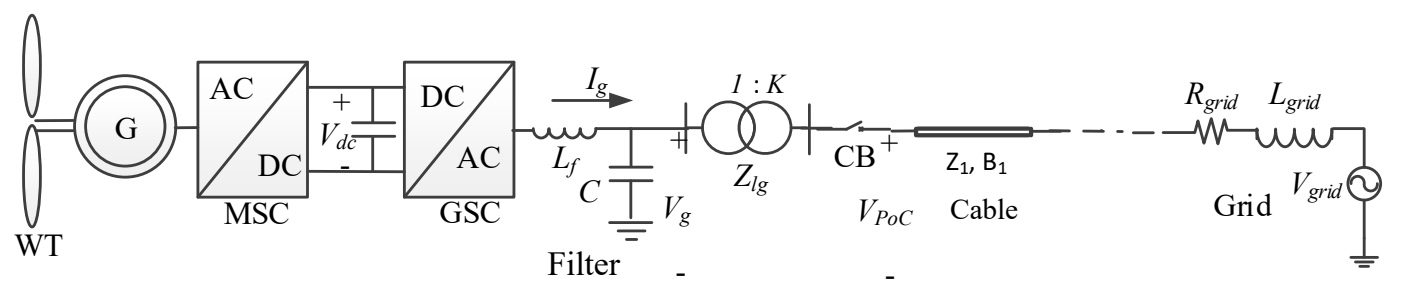

Figure 1. Simplified representation of full-scale converter-based wind turbine connected to the network.

Figure 2 shows the grid side converter of a wind turbine with its control in the dq frame. The grid side converter is a voltage source converter, and its control consists of four main parts: grid synchronization, dc-link voltage controller, current controller and pulse width modulation (PWM) [28]. The grid synchronization loop estimates the phase angle of the point-of-connection $(\mathrm{PoC})$ voltage, and this angle is used to transform the signals between the abc frame and the dq frame [29]. The current references $\left(I_{d}{ }^{r e f}\right.$ and $\left.I_{q}{ }^{r e f}\right)$ are generated from the dc-link voltage and reactive power controller. The error of these references is compared to the measured grid currents $\left(I_{d}\right.$ and $\left.I_{q}\right)$ and fed to proportional integral (PI) controllers $\left(\mathrm{G}_{\mathrm{cc}}\right)$.

The outputs of the current controllers are fed to the PWM modulator to generate the final switching signals of the insulated-gate bipolar transistors (IGBTs). The time-delay of the PWM and the discrete control can be modelled by using the Padé approximation [30]. 

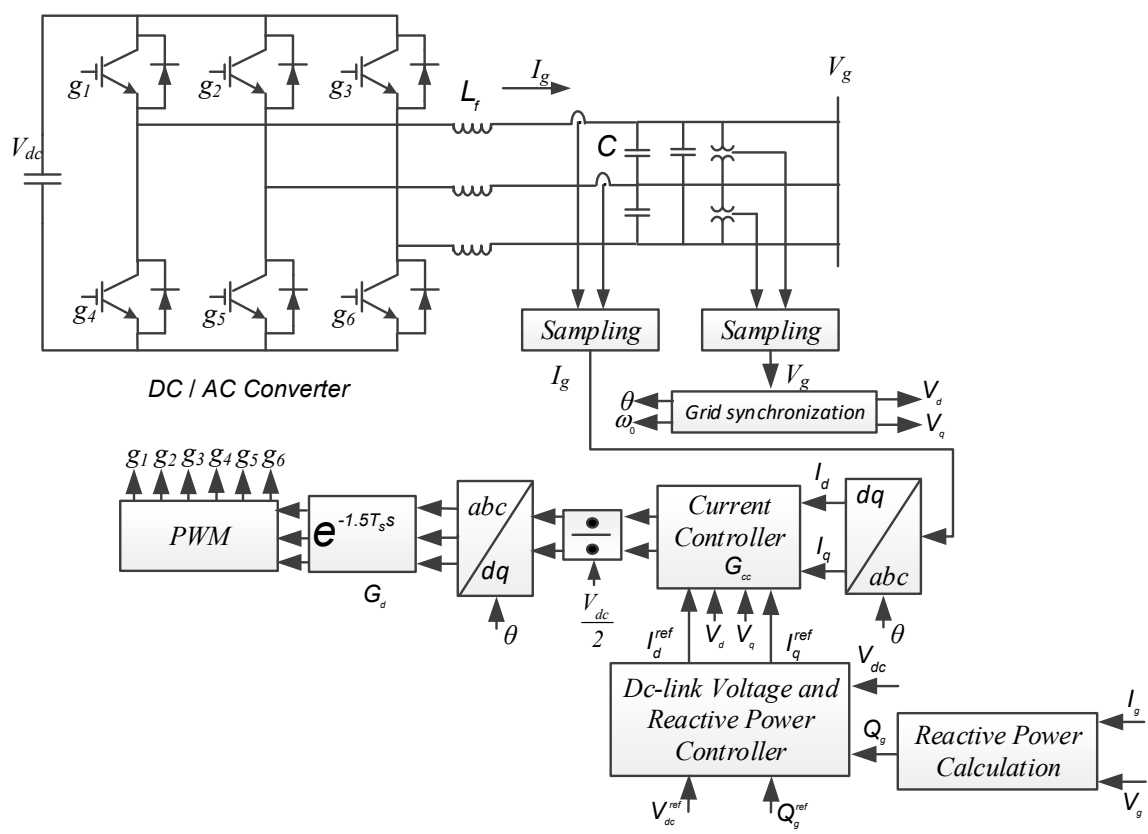

Figure 2. Grid side converter and its control, as typically used in wind turbines.

\section{Small-Signal Admittance Model in dq-Domain}

Figure 3 shows the model of the current controller, the feedforward terms, the PWM modulator and the filter choke of the grid side converter in the $\mathrm{dq}$ frame. The measurement delay is very small compared to the PWM delay and can be neglected. An outer dc-link voltage control loop is implemented in the GSC and is responsible to keep the dc-link voltage constant. The bandwidth of this outer control loop is very low compared to the fundamental frequency. This paper focuses on the oscillations around and above the fundamental frequency. Therefore, such low-bandwidth outer loops can be neglected [13]. As it is assumed that the dc-link voltage is constant, the dynamics of the dc-link controller have been neglected. Because of the dynamics of the grid synchronization loop, the dq domain model comprises a fixed global frame and a local controller frame. In the model, the measurement delay is neglected, but the PWM delay $\left(G_{d}\right)$ is included as defined in [30]. The grid voltage is added to the output current controller $\left(G_{c c}\right)$ through a high-pass/low-pass filter $\left(G_{f}\right)$.

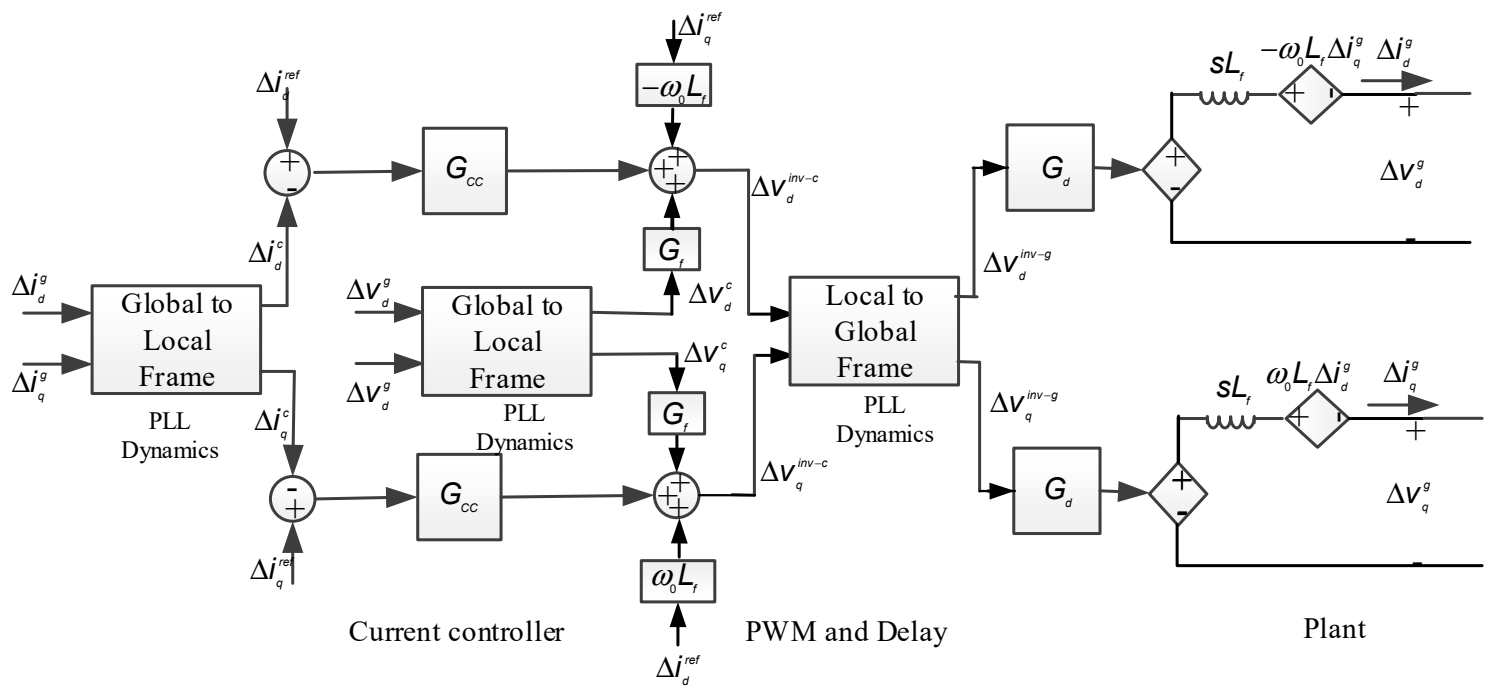

Figure 3. Dq small signal model of the current controller, PLL and the filter inductance $\left(\mathrm{L}_{\mathrm{f}}\right)$. 
According to Figure 3, the small signal output currents and dq output voltage can be obtained by the following matrices:

$$
\begin{gathered}
{\left[\begin{array}{cc}
s L_{f} & -\omega_{0} L_{f} \\
+\omega_{0} L_{f} & s L_{f}
\end{array}\right]\left[\begin{array}{c}
\Delta i_{d}^{g} \\
\Delta i_{q}^{g}
\end{array}\right]=\left[\begin{array}{cc}
G_{d} & 0 \\
0 & G_{d}
\end{array}\right]\left[\begin{array}{c}
\Delta v_{d}^{i n v-g} \\
\Delta v_{q}^{i n v-g}
\end{array}\right]+\left[\begin{array}{cc}
-1 & 0 \\
0 & -1
\end{array}\right]\left[\begin{array}{c}
\Delta v_{d}^{g} \\
\Delta v_{q}^{g}
\end{array}\right]} \\
{\left[\begin{array}{c}
\Delta v_{d}^{i n v-c} \\
\Delta v_{q}^{i n v-c}
\end{array}\right]=\left[\begin{array}{cc}
-G_{c c} & 0 \\
0 & -G_{c c}
\end{array}\right]\left[\begin{array}{l}
\Delta i_{d}^{c} \\
\Delta i_{q}^{c}
\end{array}\right]+\left[\begin{array}{cc}
G_{f} & 0 \\
0 & G_{f}
\end{array}\right]\left[\begin{array}{c}
\Delta v_{d}^{c} \\
\Delta v_{q}^{c}
\end{array}\right]}
\end{gathered}
$$

By neglecting the dynamics of the grid synchronization loop, e.g., PLL, the dq frame of the plant and the $\mathrm{dq}$ frame of the controller would be the same, i.e.,

$$
\left[\begin{array}{l}
\Delta i_{d}^{c} \\
\Delta i_{q}^{c}
\end{array}\right]=\left[\begin{array}{c}
\Delta i_{d}^{g} \\
\Delta i_{q}^{g}
\end{array}\right]=\left[\begin{array}{l}
\Delta i_{d} \\
\Delta i_{q}
\end{array}\right]\left[\begin{array}{l}
\Delta V_{d}^{c} \\
\Delta V_{q}^{c}
\end{array}\right]=\left[\begin{array}{l}
\Delta V_{q}^{g} \\
\Delta V_{q}^{g}
\end{array}\right]=\left[\begin{array}{c}
\Delta V_{d} \\
\Delta V_{q}
\end{array}\right]\left[\begin{array}{c}
\Delta V_{d}^{i n v-g} \\
\Delta V_{q}^{i n v-g}
\end{array}\right]=\left[\begin{array}{c}
\Delta V_{d}^{i n v-c} \\
\Delta V_{q}^{i n v-c}
\end{array}\right]=\left[\begin{array}{c}
\Delta V_{d}^{i n v} \\
\Delta V_{q}^{i n v}
\end{array}\right]
$$

By substituting (2) and (3) in (1), the admittance matrix can be obtained as

$$
-\left[\begin{array}{c}
\Delta i_{d} \\
\Delta i_{q}
\end{array}\right]=Y_{d q}\left[\begin{array}{c}
\Delta v_{d} \\
\Delta v_{q}
\end{array}\right] \Rightarrow Y_{d q}=-\left[\begin{array}{cc}
s L_{f}+G_{d} G_{c c} & -\omega_{0} L_{f} \\
+\omega_{0} L_{f} & s L_{f}+G_{d} G_{c c}
\end{array}\right]^{-1}\left[\begin{array}{cc}
G_{d} G_{f}-1 & 0 \\
0 & G_{d} G_{f}-1
\end{array}\right]
$$

where the dynamics of the grid synchronization loop are ignored.

For the grid synchronization loop, the synchronous reference frame phase-locked loop (SRF-PLL) can be used and the small signal model is shown in Figure 4.

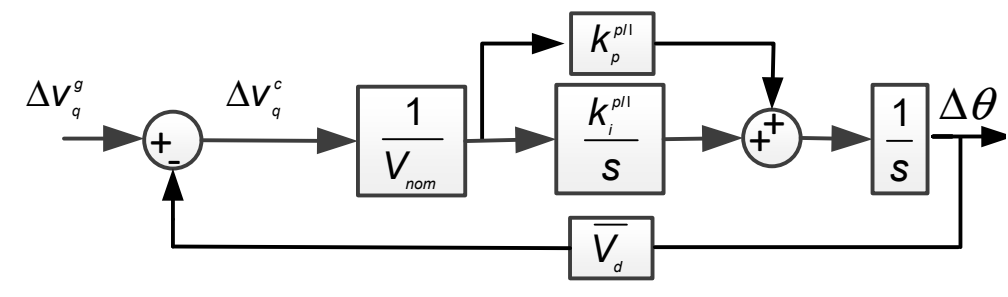

(a)

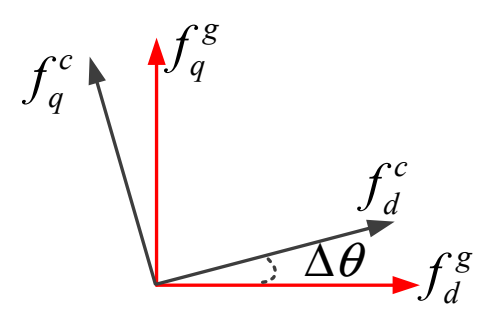

(b)

Figure 4. Synchronous reference frame phase-locked loop (SRF-PLL), (a) small signal model of PLL, (b) controller $\left(f_{d q}{ }^{c}\right)$ and system $\left(f_{d q}{ }^{g}\right)$ frames under small-signal perturbations because of the PLL dynamics.

When small-signal perturbations are added to the $V_{g}$ voltage, the estimated angle will have a small error $(\Delta \theta)$ between the local (controller) and global (plant) frames because of the PLL dynamics. In Figure $4 \mathrm{~b}, f_{d q}{ }^{c}$ corresponds to the estimated angle and $f_{d q}{ }^{g}$ corresponds to the actual angle. Based on Figure 4, the voltage and current in the controller frame and plant frame under small-signal perturbations can be derived as

$$
\begin{aligned}
& {\left[\begin{array}{l}
\Delta i_{d}^{c} \\
\Delta i_{q}^{c}
\end{array}\right]=\left[\begin{array}{l}
\Delta i_{d}^{g} \\
\Delta i_{q}^{g}
\end{array}\right]+\left[\begin{array}{cc}
0 & G_{p l l} \bar{I}_{q} \\
0 & -G_{p l l} \bar{I}_{d}
\end{array}\right]\left[\begin{array}{c}
\Delta V_{d}^{g} \\
\Delta V_{q}^{g}
\end{array}\right]\left[\begin{array}{l}
\Delta V_{d}^{c} \\
\Delta V_{q}^{c}
\end{array}\right]=\left[\begin{array}{c}
\Delta V_{d}^{g} \\
\Delta V_{q}^{g}
\end{array}\right]+\left[\begin{array}{cc}
0 & G_{p l l} \bar{V}_{q} \\
0 & -G_{p l l} \bar{V}_{d}
\end{array}\right]\left[\begin{array}{c}
\Delta V_{d}^{g} \\
\Delta V_{q}^{g}
\end{array}\right]} \\
& {\left[\begin{array}{l}
\Delta V_{d}^{i n v-g} \\
\Delta V_{q}^{i n v-g}
\end{array}\right]=\left[\begin{array}{l}
\Delta V_{d}^{i n v-c} \\
\Delta V_{q}^{i n v-c}
\end{array}\right]+\left[\begin{array}{cc}
0 & -G_{p l l} \bar{V}_{q}^{i n v} \\
0 & G_{p l l} \bar{V}_{d}^{i n v}
\end{array}\right]\left[\begin{array}{c}
\Delta V_{d}^{g} \\
\Delta V_{q}^{g}
\end{array}\right]}
\end{aligned}
$$


where $\overline{I_{d}}, \overline{I_{q}}, \overline{V_{d}}, \overline{V_{q}}, \bar{V}_{d}^{i n v}$ and $\bar{V}_{q}^{i n v}$ are the operating point values of the converter. $\Delta \theta$ can be derived as

$$
\Delta \theta=G_{p l l} \Delta V_{q}^{g}=\frac{G_{P I}^{P L L}}{V_{n o m}+G_{P I}^{P L L} \bar{V}_{d}} \Delta V_{q}^{g} \quad G_{P I}^{P L L}=K_{p}^{p l l}+\frac{K_{i}^{p l l}}{s}
$$

Using (1), (2), (5) and (6) and some simplifications, the small signal admittance matrix can be obtained by

$$
\begin{aligned}
& Y_{d q}=\left[\begin{array}{ll}
y_{d d}(s) & y_{d q}(s) \\
y_{q d}(s) & y_{q q}(s)
\end{array}\right]=-\left[\begin{array}{c}
\Delta V_{d}^{g} \\
\Delta V_{q}^{g}
\end{array}\right]^{-1} \times\left[\begin{array}{c}
\Delta i_{d}^{g} \\
\Delta i_{q}^{g}
\end{array}\right]=-\left[\begin{array}{cc}
s L_{f}+G_{c c} G_{d} & -\omega_{0} L_{f} \\
+\omega_{0} L_{f} & s L_{f}+G_{c c} G_{d}
\end{array}\right]^{-1} \\
& \times\left[\begin{array}{cc}
G_{f} G_{d}-1 & -G_{c c} G_{d} G_{p l l} \bar{I}_{q}+G_{f} G_{d} G_{p l l} \bar{V}_{q}-G_{p l l} G_{d} \bar{V}_{q}^{i n v} \\
0 & G_{c c} G_{d} G_{p l l} \bar{I}_{d}+G_{f} G_{d}-G_{f} G_{d} G_{p l l} \bar{V}_{d}+G_{p l l} G_{d} \bar{V}_{d}^{i n v}-1
\end{array}\right]
\end{aligned}
$$

\section{Equivalent Small-Signal Admittance Model in Sequence Domain}

In the previous section, the small-signal admittance model of the converter in the dq domain has been obtained. However, the admittance model can also be obtained in the positive-negative sequence domain $[18,20]$. The small-signal admittance matrix in the sequence domain can also be written by a $2 \times 2$ admittance matrix, where its off-diagonal elements show a coupling between the positive and negative components. This coupling is very small and can be neglected [7]. The relationship between the sequence domain admittance definition and dq domain admittance definition [20] can be obtained by the following equation as derived in Appendix A.

$$
\left[\begin{array}{ll}
y_{p p}(s) & y_{p n}(s) \\
y_{n p}(s) & y_{n n}(s)
\end{array}\right]=\left[\begin{array}{cc}
1 & 1 \\
-j & j
\end{array}\right]^{-1}\left[\begin{array}{ll}
y_{d d}(s) & y_{d q}(s) \\
y_{q d}(s) & y_{q q}(s)
\end{array}\right]\left[\begin{array}{cc}
1 & 1 \\
-j & j
\end{array}\right]
$$

The detailed form of Equation (8) is obtained by substituting Equation (7). In this paper, the dq domain admittance is obtained, and then the equivalent sequence admittance is derived based on (8). For the linear stability analysis of the wind power plant in the sequence domain, the coupling terms may be neglected, and the conventional positive impedance is calculated as:

$$
Z_{p}(s)=\frac{1}{y_{p p}\left(s-j \omega_{1}\right)}
$$

\section{Total Wind Turbine Thévenin Impedance}

As shown in Figure 5, there is a filter capacitor and a transformer in the output circuit of the wind turbine. Therefore, the total Thévenin impedance of the wind turbine can be equated to

$$
Z_{W}=K^{2}\left[\left(\frac{Z_{c f} Z_{p}}{Z_{C f}+Z_{p}}\right)+Z_{L g}\right]=K^{2} \frac{Z_{C f} Z_{p}+Z_{L g} Z_{p}+Z_{C f} Z_{L g}}{Z_{C f}+Z_{p}}
$$

where $Z_{p}$ is the equivalent small-signal positive impedance of the wind turbine, $Z_{c f}$ is the filter capacitor impedance, $Z_{l g}$ is the leakage impedance transformed in the primary side, and $K$ is the transformer voltage ratio. As can be seen, $Z_{p}$ is in parallel with $Z_{c f}$ and they are in series with $Z_{l g}$. Then this equivalent impedance is transferred to the high-voltage side by the transformer ratio to the power of two $\left(K^{2}\right)$. 


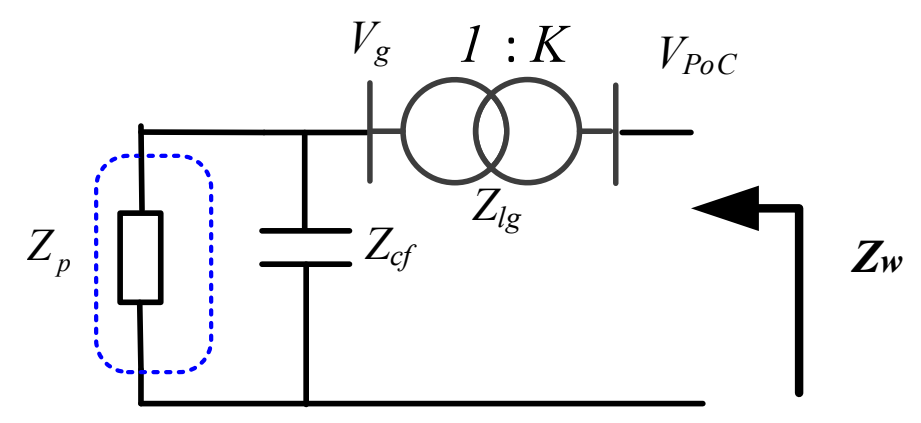

Figure 5. Simplified wind turbine (WT) system model, $Z_{p}=$ equivalent small-signal positive impedance, $Z_{c f}=$ filter capacitor impedance, $Z_{l g}=$ leakage impedance.

\section{Case Study of a Wind Power Plant Connected to a Weak Grid}

In order to perform an impedance-based stability analysis, an aggregated model of the wind power plant is required. For example, Figure 6 shows a $108 \mathrm{MW}$ wind power plant with three strings and ten 3.6 MW wind turbines installed on each string. All wind turbines in the wind power plant are assumed to have the same structure. Therefore, the impedance equivalent of the aggregated wind turbines can be obtained based on per-unit values.

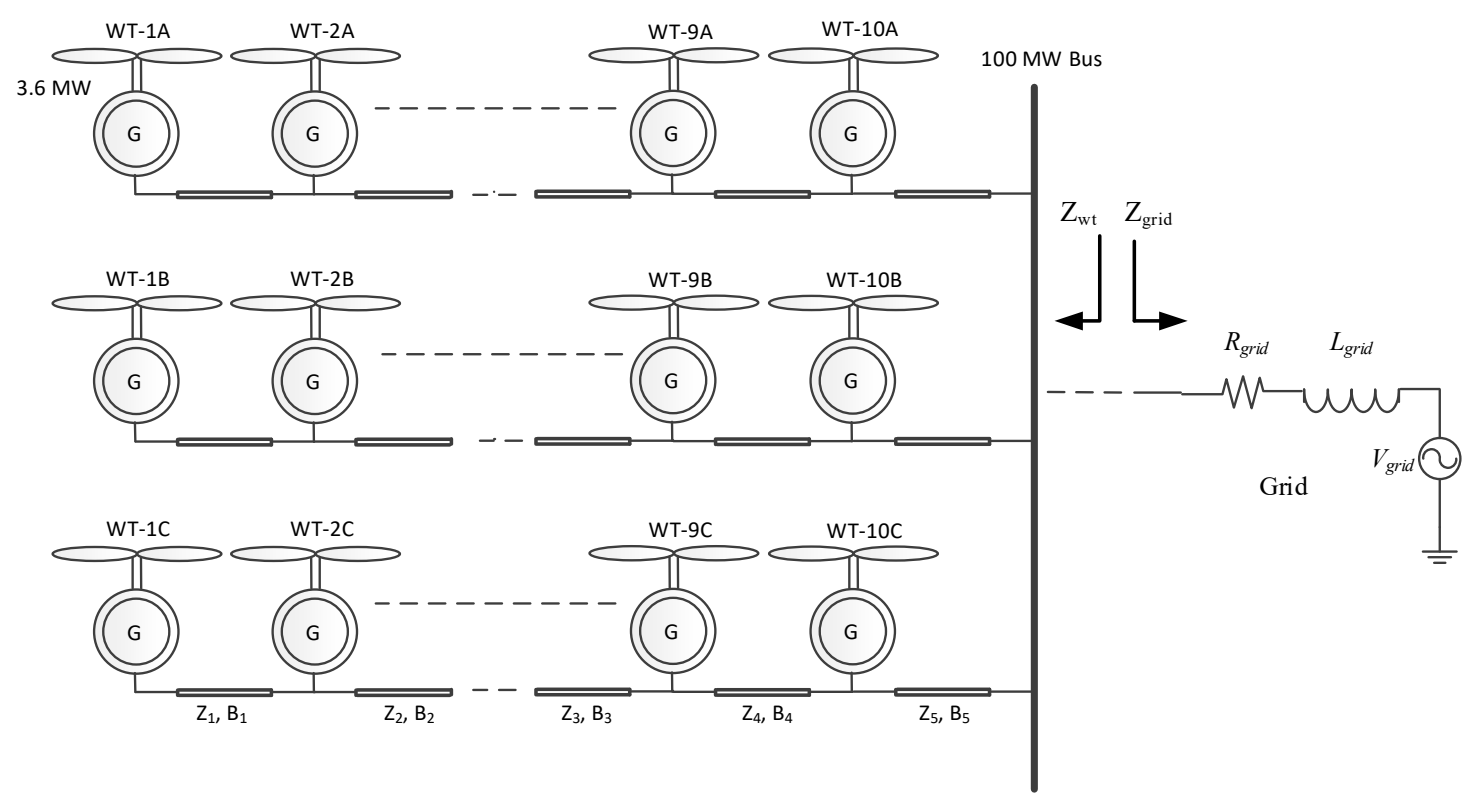

Figure 6. A $108 \mathrm{MW}$ wind power plant, including three $36 \mathrm{MW}$ strings.

If it is assumed that the voltages and currents of the wind turbines are the same, ten 3.6 MW wind turbines of each feeder can be aggregated into one $36 \mathrm{MW}$ wind turbine (see Figure 7), where its equivalent series cable parameter can be obtained $[31,32]$ by

$$
Z_{A T}=\frac{\sum_{m=1}^{10} m^{2} Z_{m}}{10^{2}} \quad B_{A T}=\sum_{m=1}^{10} B_{m}
$$




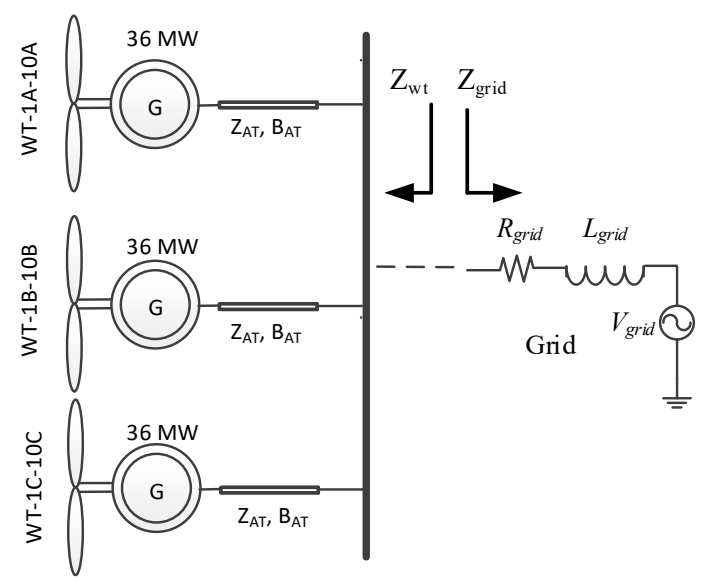

(a)

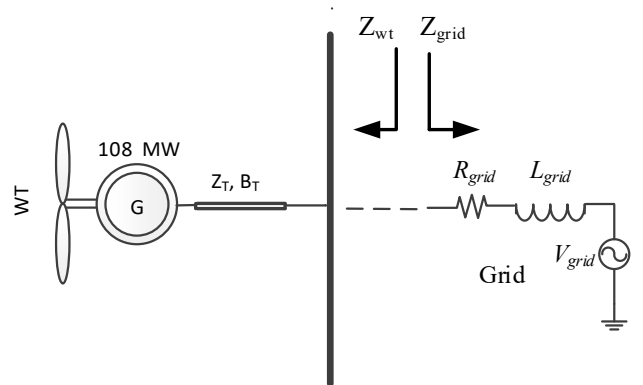

(b)

Figure 7. Aggregated model of the $108 \mathrm{MW}$ wind power plant shown in Figure 8, (a) aggregated model on each string as one $36 \mathrm{MW}$ wind turbine, (b) aggregated model of three strings into one $108 \mathrm{MW}$ wind turbine.

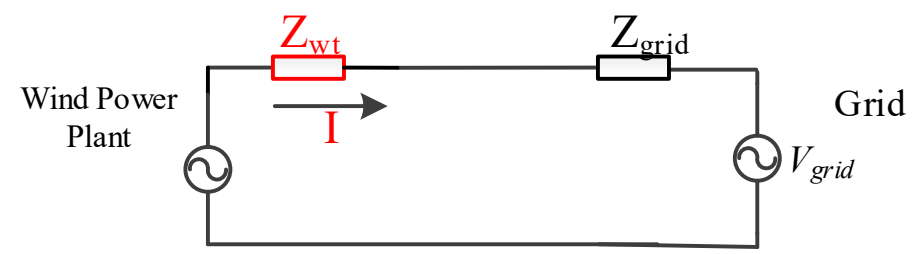

Figure 8. Aggregated wind power plant model connected to weak grid.

$\mathrm{Z}_{\mathrm{AT}}$ is the equivalent series impedance, and $\mathrm{B}_{\mathrm{AT}}$ is the equivalent shunt susceptance, respectively. Finally, three $36 \mathrm{MW}$ parallel aggregated wind turbines can be aggregated as one $108 \mathrm{MW}$ wind turbine (shown in Figure 7), where its equivalent series parameter can be calculated by

$$
Z_{T}=\frac{Z_{A T}}{3} \quad B_{T}=3 B_{A T}
$$

\subsection{Stability Analysis}

By obtaining the equivalent wind power plant impedance and the equivalent grid impedance, Figure 8 can be used for stability analysis. Based on this figure, the transfer function for the current can be obtained as

$$
I(s)=\frac{V_{w t}-V_{\text {grid }}}{Z_{w t}+Z_{\text {grid }}}=\left(\frac{V_{w t}-V_{\text {grid }}}{Z_{w t}}\right)\left(\frac{1}{1+Z_{\text {grid }} / Z_{w t}}\right)
$$

For the stability analysis, the poles of the system can be obtained by solving the following equation:

$$
Z_{w t}+Z_{\text {grid }}=0
$$

As the wind power plant is stable when connected to a strong grid, i.e., $V_{w t} / Z_{w t}$ has no right-half plane poles, an alternative option for the stability analysis is to assess the Nyquist stability criterion for $Z_{\text {grid }} / Z_{w w}$.

A weak grid model is considered here including inductive reactance $\left(X_{g}\right)$, resistance $(R)$, and series capacitive reactance $\left(X_{c}\right)$ for compensation. To illustrate sensitivity to variation in the network, short-circuit level, $X_{g} / R$ ratio and line series compensation, three scenarios, are studied, all based on a fictitious wind turbine converter controller, the parameters of which are listed in [21]. The wind turbine has been tuned for a strong grid. In Appendix B, all the variables and parameters used in the figures are summarized. 
6.1.1. Short Circuit Ratio (SCR) Variations for $X_{g} / R=20$ and $X_{c} / X_{g}=0$

In this scenario, the SCR is changed from 1.25 to $1.5, X_{g} / R=20$ and $X_{c} / X_{g}=0$ (without series compensation). Some poles of the system are shown in Table 1 . As it can be seen, there is an unstable pole for SCR $=1.25$.

Table 1. Poles for short circuit ratio (SCR) variations for $X_{g} / R=20$ and $X_{c} / X_{g}=0$.

\begin{tabular}{cccc}
\hline SCR & $\boldsymbol{P}_{\mathbf{1}}$ & $\boldsymbol{P}_{\mathbf{2}}$ & $\boldsymbol{P}_{\mathbf{3}}$ \\
\hline 1.25 & $123+j 184$ & $4.61+j 449$ & $-240+j 596$ \\
\hline 1.5 & $-117+j 189$ & $-30.6+j 446$ & $-297+j 669$ \\
\hline
\end{tabular}

Nyquist criterion-based stability analyses for SCR $=1.25$ and SCR $=1.5$ are shown in Figure 9. When the SCR $=1.25$, there is an encirclement of the point -1 , which shows that the system is unstable.

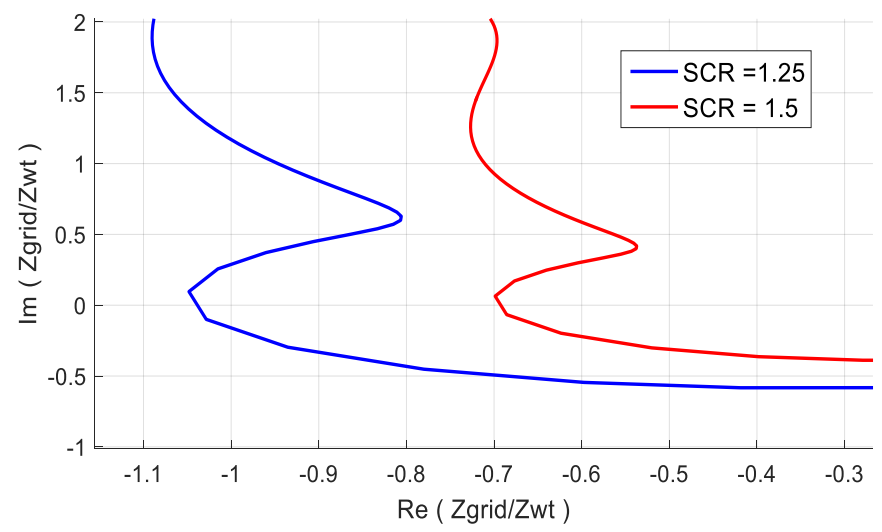

Figure 9. Nyquist criteria under SCR variations for $X_{g} / R=20$ and $X_{c} / X_{g}=0$.

6.1.2. $X_{g} / R$ variations for $S C R=1.25$ and $X_{c} / X_{g}=0$

In this scenario, $X_{g} / R$ is decreased from 20 to 5 and to 2.5 , when the $S C R=1.25$. As it can be seen from the pole analysis in Table 2 and the Nyquist analysis in Figure 10, the system becomes more stable by decreasing the $X_{g} / R$ ratio.

Table 2. Poles for $X_{g} / R$ variations for $S C R=1.25$ and $X_{c} / X_{g}=0$.

\begin{tabular}{cccc}
\hline $\boldsymbol{X}_{\boldsymbol{g}} / \boldsymbol{R}$ & $\boldsymbol{P}_{\mathbf{1}}$ & $\boldsymbol{P}_{\mathbf{2}}$ & $\boldsymbol{P}_{\mathbf{3}}$ \\
\hline 20 & $-123+j 184$ & $4.61+j 449$ & $-240+j 596$ \\
\hline 5 & $-124+j 189$ & $2.07+j 438$ & $-255+j 596$ \\
\hline 2.5 & $-125+j 196$ & $-2.97+j 427$ & $-245+j 596$ \\
\hline
\end{tabular}




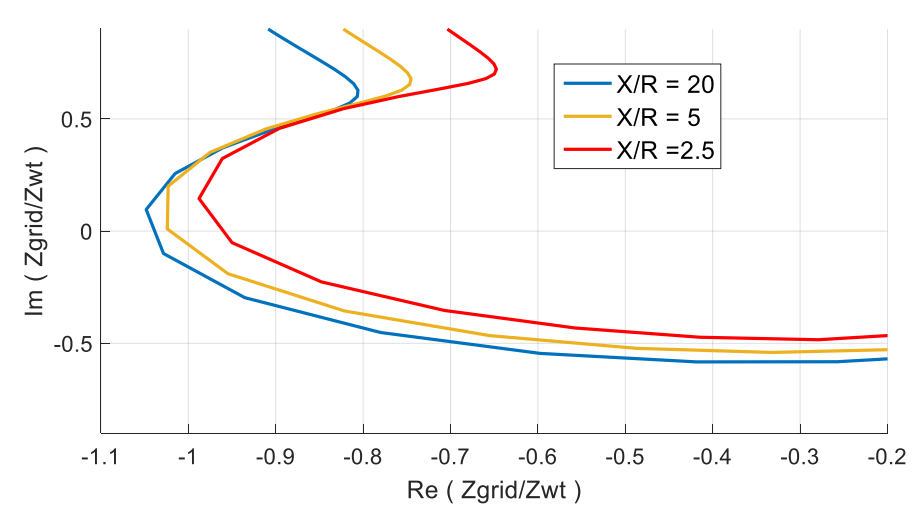

Figure 10. Nyquist criteria under $X_{g} / R$ variations for $S C R=1.25$ and $X_{c} / X_{g}=0$.

6.1.3. $X_{c} / X_{g}$ Variations for $S C R=1.25$ and $X_{g} / R=20$

Table 3 and Figure 11 show the corresponding poles and Nyquist criteria under $X_{c} / X_{g}$ variations for $\mathrm{SCR}=1.25$ and $X_{g} / R=20$. It can be seen that the real part of the pole around $70 \mathrm{~Hz}$ becomes more negative (more stable) along with more compensation.

Table 3. Poles for $X_{c} / X_{g}$ variations for $S C R=1.25$ and $X_{g} / R=20$.

\begin{tabular}{cccc}
\hline $\boldsymbol{X}_{\boldsymbol{c}} / \boldsymbol{X}_{\boldsymbol{g}}$ & $\boldsymbol{P}_{\mathbf{1}}$ & $\boldsymbol{P}_{\mathbf{2}}$ & $\boldsymbol{P}_{\mathbf{3}}$ \\
\hline 0 & $-123+j 184$ & $4.61+j 449$ & $-240+j 596$ \\
\hline 0.1 & $-119+j 182$ & $-0.61+j 449$ & $-239+j 600$ \\
\hline 0.3 & $-110+j 175$ & $-11.1+j 450$ & $-237+j 609$ \\
\hline
\end{tabular}

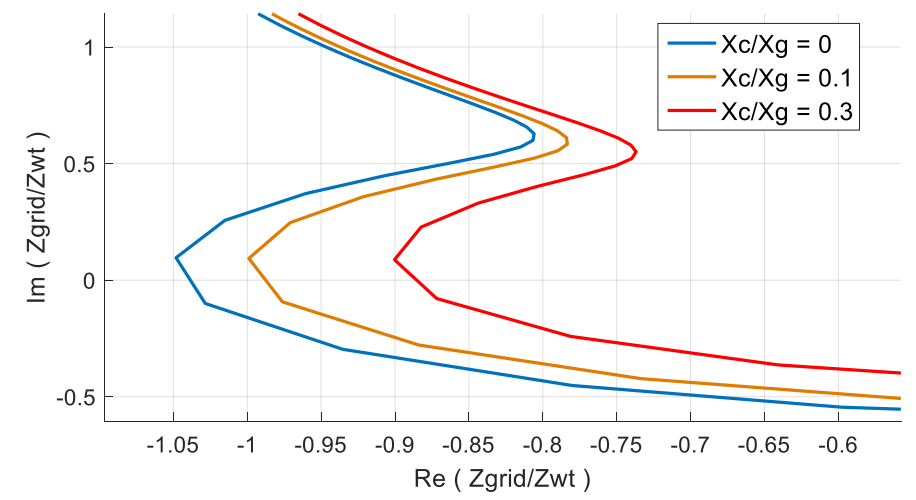

Figure 11. Nyquist criteria under $X_{c} / X_{g}$ variations for $S C R=1.25$ and $X / R=$ 206.1.4. Time-domain simulation result.

In order to validate the frequency-domain analysis, a time-domain simulation was performed for $\mathrm{SCR}=1.25, \mathrm{Xg} / \mathrm{R}=20$ and $\mathrm{X}_{c} / \mathrm{X}_{g}=0$. The current injected to the grid and its fast Fourier transform (FFT) are shown in Figure 12. As it can be seen, some oscillations around $73 \mathrm{~Hz}$ (459 rad) are increased until the wind turbine goes to trip. This result is matched with the frequency-domain result in Table 1 , which indicates there is one unstable mode as $4.61+j 449$. 


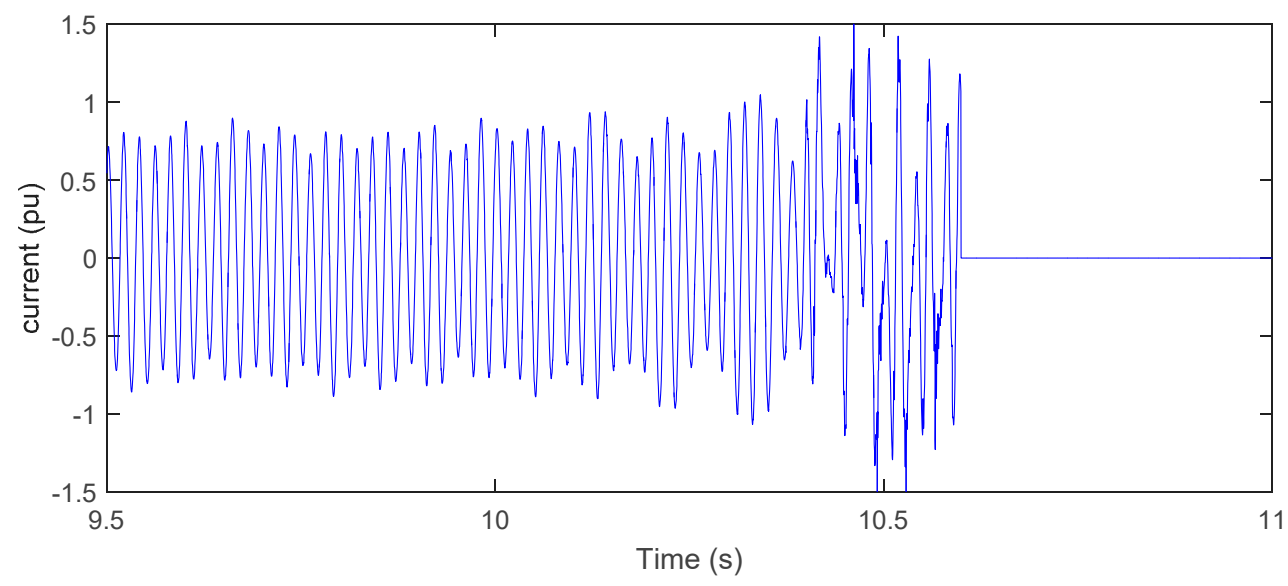

(a)

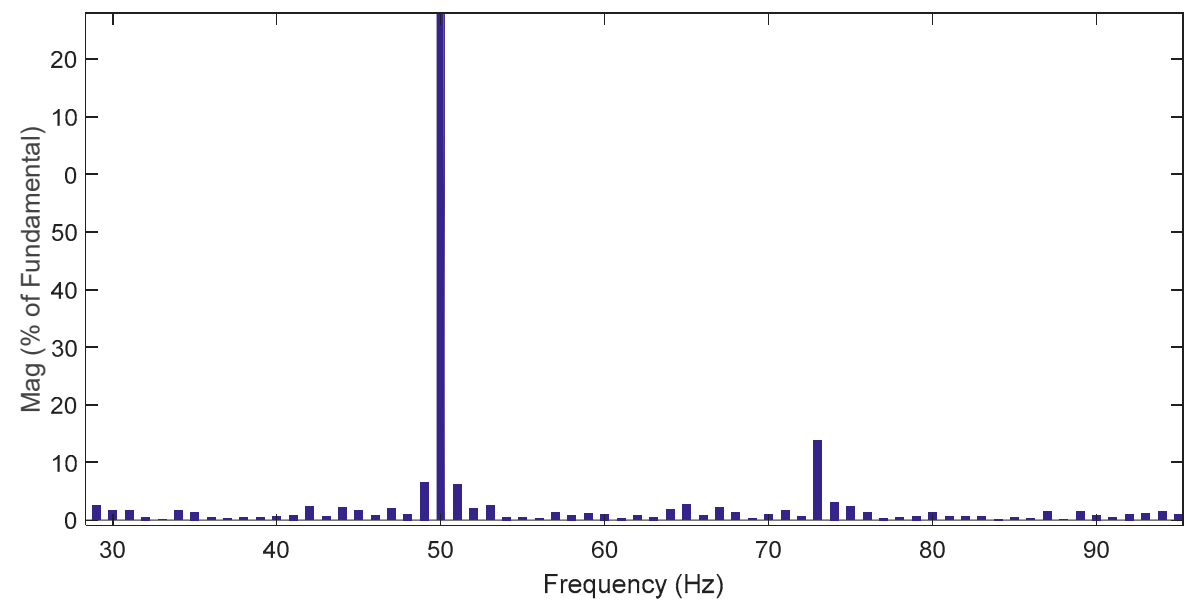

(b)

Figure 12. Time-domain simulation for $\mathrm{SCR}=1.25, X_{g} / R=20$ and $X_{\mathcal{c}} / X_{g}=0$ (an unstable case), (a) current, (b) fast Fourier transform (FFT) of the current before trip.

\section{Conclusions}

This paper presents a unified impedance-based modelling and analysis tool to predict unstable conditions resulting from the interactions between the wind power plant and the weak grid. The proposed approach allows to have advantages of both the dq domain modelling and sequence domain modelling. This enables transmission system operators (TSOs) to do stability analyses of large power systems more efficiently, and it enables wind turbine manufacturers to optimise the controller parameters to increase the stability margin under a weak grid connection. A $108 \mathrm{MW}$ wind power plant has been studied and the short circuit ratio (SCR) of the grid is considered to be 1.25 and 1.5. The poles of the closed-loop system in the frequency-domain are calculated to find the frequency and damping of the oscillatory modes. Nyquist criteria of the open-loop system are plotted to predict easily the stability margin of the wind power plant connected to the weak grid. The results of a simulated wind power plant have shown that a wind power plant which is tuned for a strong grid can become unstable when connected to a weak grid. The proposed method is a valuable tool when adapting and retuning the control system to actual grid conditions.

Author Contributions: E.E. and F.B. proposed the initial methodology, T.L. proposed how to apply the method for weak grids, J.G.N. provided the data, and P.C.K. reviewed and edited the paper.

Funding: This research was funded by "Vestas Wind System A/S" and "Innovation Fund Denmark, grant number 8054-00023B".

Conflicts of Interest: The authors declare no conflict of interest. 


\section{Appendix A}

A Relationship between dq-domain admittance/impedance and sequence-domain admittance/impedance.

In this part, the relationship between dq-domain admittance/impedance and sequence-domain admittance/impedance is derived.

Following Park's transformation is used to convert three-phase variables into a dq reference frame:

$$
\left[\begin{array}{l}
X_{d} \\
X_{q}
\end{array}\right]=\frac{2}{3}\left[\begin{array}{ccc}
\cos (\theta) & \cos \left(\theta-\frac{2 \pi}{3}\right) & \cos \left(\theta+\frac{2 \pi}{3}\right) \\
-\sin (\theta) & -\sin \left(\theta+\frac{2 \pi}{3}\right) & -\sin \left(\theta+\frac{2 \pi}{3}\right)
\end{array}\right]\left[\begin{array}{c}
X_{a} \\
X_{b} \\
X_{c}
\end{array}\right]
$$

For $\theta=\omega_{1} t$ in (A1), three-phase perturbed voltages described in (29) can be transformed to the dq-domain as

$$
\begin{aligned}
& {\left[\begin{array}{c}
\Delta v_{d}+\bar{V}_{d} \\
\Delta v_{q}+\bar{V}_{q}
\end{array}\right]=\frac{2}{3}\left[\begin{array}{ccc}
\cos \left(2 \pi f_{1} t\right) & \cos \left(2 \pi f_{1} t-\frac{2 \pi}{3}\right) & \cos \left(2 \pi f_{1} t+\frac{2 \pi}{3}\right) \\
-\sin \left(2 \pi f_{1} t\right) & -\sin \left(2 \pi f_{1} t+\frac{2 \pi}{3}\right) & -\sin \left(2 \pi f_{1} t+\frac{2 \pi}{3}\right)
\end{array}\right] \times} \\
& {\left[\begin{array}{c}
V_{1} \cos \left(2 \pi f_{1} t+\phi_{v 1}\right)+\hat{v}_{p} \cos \left[2 \pi\left(f_{p}+f_{1}\right) t+\phi_{v p}\right]+\hat{v}_{n} \cos \left[2 \pi\left(f_{p}-f_{1}\right) t+\phi_{v n}\right] \\
V_{1} \cos \left(2 \pi f_{1} t+\phi_{v 1}-\frac{2 \pi}{3}\right)+\hat{v}_{p} \cos \left[2 \pi\left(f_{p}+f_{1}\right) t+\phi_{v p}-\frac{2 \pi}{3}\right]+\hat{v}_{n} \cos \left[2 \pi\left(f_{p}-f_{1}\right) t+\phi_{v n}+\frac{2 \pi}{3}\right] \\
V_{1} \cos \left(2 \pi f_{1} t+\phi_{v 1}+\frac{2 \pi}{3}\right)+\hat{v}_{p} \cos \left[2 \pi\left(f_{p}+f_{1}\right) t+\phi_{v p}+\frac{2 \pi}{3}\right]+\hat{v}_{n} \cos \left[2 \pi\left(f_{p}-f_{1}\right) t+\phi_{v n}-\frac{2 \pi}{3}\right]
\end{array}\right]}
\end{aligned}
$$

Based on trigonometric formulas and some simplification, (A2) can be simplified as

$$
\begin{aligned}
& {\left[\begin{array}{c}
\Delta v_{d}+\bar{V}_{d} \\
\Delta v_{q}+\bar{V}_{q}
\end{array}\right]=\left[\begin{array}{c}
V_{1} \cos \left(\phi_{v 1}\right)+\hat{v}_{p} \cos \left(2 \pi \mathrm{f}_{p} t+\phi_{v p}\right)+\hat{v}_{n} \cos \left(2 \pi \mathrm{f}_{p} t+\phi_{v n}\right) \\
V_{1} \sin \left(\phi_{v 1}\right)+\hat{v}_{p} \sin \left(2 \pi \mathrm{f}_{p} t+\phi_{v p}\right)-\hat{v}_{n} \sin \left(2 \pi \mathrm{f}_{p} t+\phi_{v n}\right)
\end{array}\right]} \\
& \quad=\left[\begin{array}{c}
V_{1} \cos \left(\phi_{v 1}\right)+\hat{v}_{p} \cos \left(2 \pi \mathrm{f}_{p} t+\phi_{v p}\right)+\hat{v}_{n} \cos \left(2 \pi \mathrm{f}_{p} t+\phi_{v n}\right) \\
V_{1} \cos \left(\phi_{v 1}-\frac{\pi}{2}\right)+\hat{v}_{p} \cos \left(2 \pi \mathrm{f}_{p} t+\phi_{v p}-\frac{\pi}{2}\right)-\hat{v}_{n} \cos \left(2 \pi \mathrm{f}_{p} t+\phi_{v n}-\frac{\pi}{2}\right)
\end{array}\right]
\end{aligned}
$$

According to (A3), small-signal dq voltages can be obtained based small-signal positive-negative voltages as

$$
\left[\begin{array}{c}
\Delta v_{d} \\
\Delta v_{q}
\end{array}\right]=\left[\begin{array}{c}
\hat{v}_{p} e^{j \phi_{v p}}+\hat{v}_{n} e^{j \phi_{v n}} \\
-j \hat{v}_{p} e^{j \phi_{v p}}+j \hat{v}_{n} e^{j \phi_{v n}}
\end{array}\right]=\left[\begin{array}{cc}
1 & 1 \\
-j & j
\end{array}\right]\left[\begin{array}{c}
\hat{v}_{p} e^{j \phi_{v p}} \\
\hat{v}_{n} e^{j \phi_{v n}}
\end{array}\right]=\left[\begin{array}{cc}
1 & 1 \\
-j & j
\end{array}\right]\left[\begin{array}{c}
\Delta v_{p} \\
\Delta v_{n}
\end{array}\right]
$$

The same relationship is valid for three-phase currents.

$$
\left[\begin{array}{l}
\Delta i_{d} \\
\Delta i_{q}
\end{array}\right]=\left[\begin{array}{cc}
1 & 1 \\
-j & j
\end{array}\right]\left[\begin{array}{c}
\Delta i_{p} \\
\Delta i_{n}
\end{array}\right]
$$

As already presented in this paper, the small-signal behaviour of a three-phase converter can be modelled in dq domain by

$$
\left[\begin{array}{l}
\Delta i_{d} \\
\Delta i_{q}
\end{array}\right]=\left[\begin{array}{ll}
y_{d d}(s) & y_{d q}(s) \\
y_{q d}(s) & y_{q q}(s)
\end{array}\right]\left[\begin{array}{c}
\Delta v_{d} \\
\Delta v_{q}
\end{array}\right]
$$

By substituting (A4) and (A5) in (A6) we get

$$
\left[\begin{array}{cc}
1 & 1 \\
-j & j
\end{array}\right]\left[\begin{array}{c}
\Delta i_{p} \\
\Delta i_{n}
\end{array}\right]=\left[\begin{array}{ll}
y_{d d}(s) & y_{d q}(s) \\
y_{q d}(s) & y_{q q}(s)
\end{array}\right]\left[\begin{array}{cc}
1 & 1 \\
-j & j
\end{array}\right]\left[\begin{array}{c}
\Delta v_{p} \\
\Delta v_{n}
\end{array}\right]
$$

Equation (A7) can be rewritten as

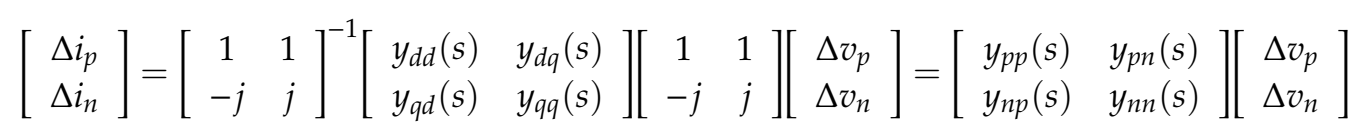


Based on (A8), the relationship between sequence-domain admittance definition and dq-domain admittance definition can be obtained by

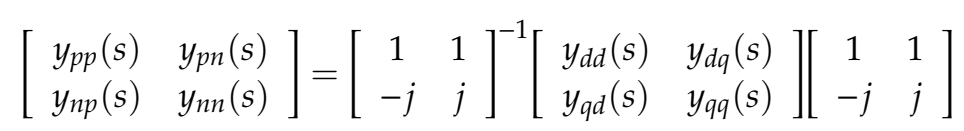

\section{Appendix B}

In the following table, all the variables and parameters used in the figures are defined.

Table A1. Definition of the variables and parameters used in the figures.

\begin{tabular}{cccc}
\hline$V_{d c}$ & dc-link voltage & $Q_{g}^{r e f}$ & reactive power reference \\
\hline$L_{f}$ & filter chock & $Q_{g}$ & injected reactive power \\
\hline$C$ & filter capacitor & $V_{d c}^{r e f}$ & dc-link voltage reference \\
\hline$Z_{\text {lg }}$ & transformer leakage impedance & $I_{d}^{r e f}$ & d-axis current reference \\
\hline$V_{g}$ & filter capacitor voltage & $I_{q}^{r e f}$ & q-axis current reference \\
\hline$I_{g}$ & wind turbine current & $T_{s}$ & sampling time \\
\hline$K$ & transformer ratio & $V_{n o m}$ & nominal voltage \\
\hline$V_{\text {PoC }}$ & point of connection voltage & $K_{p}^{p l l}$ & proportional gain of the PLL \\
\hline$Z_{1}$ & cable impedance & $K_{i}^{p l l}$ & integral gain of the PLL \\
\hline$B_{1}$ & cable susceptance & $Z_{p}$ & $\begin{array}{c}\text { equivalent small-signal positive } \\
\text { impedance of the wind turbine }\end{array}$ \\
\hline$R_{\text {grid }}$ & grid resistance & $Z_{c f}$ & filter capacitor impedance \\
\hline$Z_{g \text { rid }}$ & grid impedance & $Z_{\mathrm{lg}}$ & leakage impedance \\
\hline$V_{g r i d}$ & grid voltage & $Z_{w}$ & Thévenin impedance \\
\hline$V_{d}$ & d-axis voltage of the grid & $Z_{T}$ & equivalent series cable impedance \\
\hline$V_{q}$ & q-axis voltage of the grid & $B_{T}$ & equivalent shunt cable susceptance \\
\hline$\theta$ & grid voltage angle & $Z_{w t}$ & $\begin{array}{c}\text { Thévenin impedance of the aggregated } \\
\text { wind power plant }\end{array}$ \\
\hline$\omega_{0}$ & grid frequency & & \\
\hline
\end{tabular}

\section{References}

1. Mitra, P.; Zhang, L.; Harnefors, L. Offshore wind integration to a weak grid by VSC-HVDC links using power-synchronization control: A case study. IEEE Trans. Power Del. 2014, 29, 453-461. [CrossRef]

2. Pereira, F.J.F.; Rodrigues, J.M.; de Oliveira, K.L.M.; Araujo, D.R.R.P.; de Araujo, L.R. Simulations and analysis of distribution systems with aspects of smart grids using MICQ, RTDS and PSCAD. In Proceedings of the IEEE ISGT Conference, Sao Paulo, Brazil, 15-17 April 2013; pp. 1-8.

3. Zhang, Q.; Lu, G.; Zhang, C. Simulated study of the multiple cascade medium-voltage inverter based on PSCAD/EMTDC. In Proceedings of the IEEE ICEMS, Chiba, Japan, 13-16 November 2016; pp. 1-6.

4. Pogaku, N.; Prodanovic, M.; Green, T.C. Modeling, analysis and testing of autonomous operation of an inverter-based microgrid. IEEE Trans. Power Electron. 2007, 22, 613-625. [CrossRef]

5. Sun, J. Impedance-based stability criterion for grid-connected inverters. IEEE Trans. Power Electron. 2011, 26, 3075-3078. [CrossRef]

6. Badrzadeh, B.; Gupta, M.; Singh, N.; Petersson, A.; Max, L.; Høgdahl, M. Power system harmonic analysis in wind power plants-Part I: Study methodology and techniques. In Proceedings of the IEEE IAS, Las Vegas, NV, USA, 7-11 October 2012; pp. 1-11. 
7. Cespedes, M.; Sun, J. Impedance modeling and analysis of grid connected voltage-source converters. IEEE Trans. Power Electron. 2014, 29, 1254-1261. [CrossRef]

8. Bottrell, N.; Prodanovic, M.; Green, T.C. Dynamic stability of a microgrid with an active load. IEEE Trans. Power Electron. 2013, 28, 5107-5119. [CrossRef]

9. Singh, A.; Kaviani, A.K.; Mirafzal, B. On dynamic models and stability analysis of three-phase phasor PWM-based CSI for stand-alone applications. IEEE Trans. Ind. Electron. 2015, 62, 2698-2707. [CrossRef]

10. Coelho, E.A.A.; Cortizo, P.C.; Garcia, P.F.D. Small-signal stability for parallel-connected inverters in stand-alone AC supply systems. IEEE Trans. Ind. Appl. 2002, 38, 533-542. [CrossRef]

11. Kunjumuhammed, L.P.; Pal, B.C.; Oates, C.; Dyke, K.J. The adequacy of the present practice in dynamic aggregated modeling of wind farm systems. IEEE Trans. Sustain. Energy 2017, 8, 23-32. [CrossRef]

12. Kwon, J.; Wang, X.; Blaabjerg, F.; Bak, C.L.; Wood, A.R.; Watson, N. Linearized modeling methods of ac-dc converters for an accurate frequency response. IEEE J. Emerg. Sel. Top. Power Electron. 2017, 5, 1526-1541. [CrossRef]

13. Wang, X.; Blaabjerg, F. Harmonic stability in power electronic based power systems: Concept, modeling, and analysis. IEEE Trans. Smart Grid 2018, 10, 2858-2870. [CrossRef]

14. Wang, Y.; Wang, X.; Blaabjerg, F.; Chen, Z. Small-signal stability analysis of inverter-fed power systems using component connection method. IEEE Trans. Smart Grid 2018, 9, 5301-5310. [CrossRef]

15. Gaba, G.; Lefebver, S.; Mukhedkar, D. Comparative analysis and study of the dynamic stability of AC/DC systems. IEEE Trans. Power Syst. 1988, 3, 978-985. [CrossRef]

16. Hou, P.; Ebrahimzadeh, E.; Wang, X.; Blaabjerg, F.; Fang, J.; Wang, Y. Harmonic stability analysis of offshore wind farm with CCM. In Proceedings of the IEEE IECON Conference, Beijing, China, 29 October-1 November 2017; pp. 1-6.

17. Harnefors, L.; Bongiorno, M.; Lundberg, S. Input-Admittance Calculation and Shaping for Controlled Voltage-Source Converters. IEEE Trans. Ind. Electron. 2007, 54, 3323-3334. [CrossRef]

18. Shah, S.; Parsa, L. Impedance Modeling of Three-Phase Voltage Source Converters in DQ, Sequence, and Phasor Domains. IEEE Trans. Energy Convers. 2017, 32, 1139-1150. [CrossRef]

19. Holmes, D.G.; Lipo, T.A. Pulse Width Modulation for Power Converters: Principles and Practice; Wiley: Piscataway, NJ, USA, 2003.

20. Rygg, A.; Molinas, M.; Zhang, C.; Cai, X. A modified sequence-domain impedance definition and its equivalence to the dq-domain impedance definition for the stability analysis of AC power electronic systems. IEEE Trans. Emerg. Sel. Top. Power Electron. 2016, 4, 1383-1396. [CrossRef]

21. Ebrahimzadeh, E.; Blaabjerg, F.; Wang, X.; Bak, C.L.; Lund, T.; Andersen, G.K.; Suárez, C.G.; Berg, J. Small signal modeling of wind farms. In Proceedings of the IEEE ECCE Conference, Cincinnati, OH, USA, 1-5 October 2017; pp. 1-7.

22. Wen, B.; Boroyevich, D.; Burgos, R.; Mattavelli, P.; Shen, Z. Small signal stability analysis of three-phase AC systems in the presence of constant power loads based on measured $\mathrm{d}-\mathrm{q}$ frame impedances. IEEE Trans. Power Electron. 2015, 30, 5952-5963. [CrossRef]

23. Wen, B.; Boroyevich, D.; Burgos, R.; Mattavelli, P.; Shen, Z. Analysis of D-Q small-signal impedance of grid-tied inverters. IEEE Trans. Power Electron. 2016, 31, 675-687. [CrossRef]

24. Kunjumuhammed, L.P.; Pal, B.C.; Oates, C.; Dyke, K.J. Electrical oscillations in wind farm systems: Analysis and insight based on detailed modeling. IEEE Trans. Sustain. Energy 2016, 7, 51-62. [CrossRef]

25. Kwon, J.; Wang, X.; Blaabjerg, F.; Bak, C.L.; Wood, A.R.; Watson, N. Harmonic instability analysis of a single-phase grid-connected converter using a harmonic state-space modeling method. IEEE Trans. Ind. Appl. 2016, 52, 4188-4200. [CrossRef]

26. Zhang, Z.; Wang, F.; Wang, J.; Rodríguez, J.; Kennel, R. Nonlinear direct control for three-level NPC back-to-back converter PMSG wind turbine systems: Experimental assessment with FPGA. IEEE Trans. Ind. Inf. 2017, 13, 1172-1183. [CrossRef]

27. Ebrahimzadeh, E.; Blaabjerg, F.; Wang, X.; Bak, C.L. Bus participation factor analysis for harmonic instability in power electronics based power systems. IEEE Trans. Power Electron. 2018, 33, 10341-10351. [CrossRef]

28. Xing, P.; Fu, L.; Wang, G.; Wang, Y.; Zhang, Y. A compositive control method of low-voltage ride through for PMSG-based wind turbine generator system. IET Gen. Transm. Distrib. 2018, 12, 117-125. [CrossRef] 
29. Yu, L.; Li, R.; Xu, L. Distributed PLL-based control of offshore wind turbine connected with diode-rectifier based HVDC systems. IEEE Trans. Power Del. 2018, 33, 1328-1336. [CrossRef]

30. Wang, J.; Yan, J.D.; Jiang, L.; Zou, J. Delay-dependent stability of single-loop controlled grid-connected inverters with LCL filters. IEEE Trans. Power Electron. 2016, 31, 743-757. [CrossRef]

31. Ebrahimzadeh, E. Electrical Oscillations in Wind Power Plants: Modeling, Control, and Mitigation. Ph.D. Thesis, Aalborg University, Aalborg, Denmark, 2018.

32. Muljadi, E.; Pasupulati, S.; Ellis, A.; Kosterov, D. Method of equivalencing for a large wind power plant with multiple turbine representation. In Proceedings of the IEEE Power and Energy Society General Meeting, Pittsburgh, PA, USA, 20-24 July 2008; pp. 1-9.

(C) 2019 by the authors. Licensee MDPI, Basel, Switzerland. This article is an open access article distributed under the terms and conditions of the Creative Commons Attribution (CC BY) license (http://creativecommons.org/licenses/by/4.0/). 